\title{
Transport of Negative Hydrogen and Deuterium Ions in RF-driven Ion Sources
}

\author{
R. Gutser, D. Wünderlich, U. Fantz and the NNBI-Team \\ Max-Planck-Institut für Plasmaphysik, Euratom Association, D-85748 Garching, \\ Germany \\ E-mail: raphael.gutser@ipp.mpg.de
}

\begin{abstract}
Negative hydrogen ion sources are major components of neutral beam injection systems for plasma heating in future large-scale fusion experiments like ITER. In order to fulfill the requirements of the ITER neutral beam injection, a high-performance, large-area RF-driven ion source for negative ions is being developed at the MPI für Plasmaphysik. Negative hydrogen ions are mainly generated on a converter surface by impinging neutral particles and positive ions under the influence of magnetic fields and the plasma sheath potential. The 3D transport code TrajAn has been applied in order to obtain the total and spatially resolved extraction probabilities for $\mathrm{H}^{-}$and $\mathrm{D}^{-}$ions under identical plasma parameters and the realistic magnetic field topology of the ion source. A comparison of the isotopes shows a lower total extraction probability in case of deuterium ions, caused by different transport effect. The transport calculation shows that distortions of the spatial distributions of ion birth and extraction by the magnetic electron suppression field are present for both negative hydrogen and deuterium ions.
\end{abstract}




\section{Introduction}

High performance neutral beam injection systems for hydrogen are essential components in magnetically confined fusion experiments. Positive ion based systems with particle energies below $100 \mathrm{keV}$ are routinely used nowadays in fusion experiments like ASDEX Upgrade for heating and current drive [1]. Nevertheless, large fusion experiments, such as ITER, require significantly higher particle energies up to $1 \mathrm{MeV}$ in order to reach the central regions of the fusion plasma and to provide sufficient heating power and current drive.

While the neutralization efficiency for systems based on positive hydrogen ions decreases to unacceptable low values for with increasing energy, the neutralization efficiency of negative hydrogen or deuterium ions attains $60 \%$ for the required energy of $1 \mathrm{MeV}[2]$. This high neutralization efficiency of negative ions, related to the low binding energy $(0.75 \mathrm{eV})$ of the additional electron, causes, on the other hand, a higher loss rate of the negative ions. The current density delivered by negative ion sources is typically a factor of ten lower compared to positive ion sources. In order to achieve the required parameters, the effective extraction area is increased. At negative ion current densities of $j_{\mathrm{D}^{-}}=200 \mathrm{~A} / \mathrm{m}^{2}$ the effective extraction area will be $2000 \mathrm{~cm}^{2}$ to deliver $40 \mathrm{~A}$ of deuterium ions required for ITER. Furthermore, the ITER negative ion source must deliver beam pulses of up to 3600 seconds at a source pressure of $0.3 \mathrm{~Pa}$ while maintaining a ratio of co-extracted electron to deuterium ion current density $j_{\mathrm{e}} / j_{\mathrm{D}^{-}}<1$ [3] [4].

In 2007, the RF-driven ion source, developed at the MPI für Plasmaphysik was chosen as the reference source for the ITER neutral beam injectors [3]. Numerical models can help to enhance the understanding of the physical processes involved in the negative ion source. This is essential to optimize existing ion sources and to support the development of large sources, such as the half-size ITER source ELISE [5], which is an intermediate step between the present negative ion sources and the ITER source.

An important question arises from the different behavior of the negative ion source for the production of negative deuterium or hydrogen ions. Hydrogen operation is preferred during conditioning and for experimental studies because of the reduced level of co-extracted electrons. Additionally, there is no requirement for radiation protection, while Deuterium operation requires remote control with limited accessibility.

The comparability and transferability of results obtained in hydrogen operation to those for deuterium is therefore very important. The isotope effect impacts several physical aspects of the generation and extraction of negative ions, such as the RF-coupling, the plasma sheath characteristics [6] and the negative ion transport. Therefore, an investigation of the influence of the isotope effect on the transport of negative ions within the source by means of probabilistic ion transport will be presented in this study in order to allows a separation of the different effects.

A realistic description of the 3D magnetic field configuration of the ion extraction region was considered to be necessary for the investigation of the isotope effect. While existing transport codes [7] [8] use simplified assumptions with respect to the magnetic field, 
the transport code TrajAn (Trajectory Analysis) [9] is being developed at the MPI für Plasmaphysik to allow a realistic description of the $3 \mathrm{~d}$ magnetic field topology of the individual experiment. TrajAn has been applied to obtain numerical results of the extraction probability and current density contour for negative hydrogen and deuterium ions within the field topology and converter geometry of the RF-driven ion source. Identical plasma parameters were used for both species in order to ensure comparability and to separate ion transport effects.

A brief description of the RF-driven ion source is given in the following section, while detailed information regarding the individual components and operation parameters are available from [10]. Section 3 gives an overview of the magnetic field configuration of the ion source, while the transport code TrajAn is described in section 4. Results of the transport investigation and conclusions are presented in section 5 and 6 .

\section{The RF-driven Negative Ion Source}

The RF-driven ion source is divided into three parts: driver, expansion region and extraction region. A low pressure and low temperature plasma is generated inside the driver by a RF coil, which is connected to a $1 \mathrm{MHz}$ oscillator, rated for up to $100 \mathrm{~kW}$ $\mathrm{RF}$ power. A magnetic filter field in the expansion region is necessary to keep energetic electrons $\left(\mathrm{T}_{\mathbf{e}}>2 \mathrm{eV}\right)$ away from the regions of negative ion production in order to avoid collisional destruction. Negative ion production is governed by the surface effect: positive or neutral hydrogen plasma particles hit a converter surface, where they pick up one or more electrons. Measurements [11] show typical plasma parameters of $\mathrm{T}_{\mathbf{e}}=2$ $\mathrm{eV}$ electron temperature and $\mathrm{n}_{\mathbf{e}}=5 \cdot 10^{17} \mathrm{~m}^{-3}$ electron density in the extraction region resulting in survival lengths in the cm-range. Thus, only negative ions generated on the converter surface are able to contribute to the extracted current density, while negative ion contributions from other surfaces are not able to reach the extraction system.

To obtain the required high conversion rate, the walls are covered by a thin layer of cesium lowering the work function [12] of the surface. This is achieved by evaporating elemental cesium from a liquid cesium reservoir into the ion source.

The surface produced negative ions are initially accelerated towards the plasma volume by the plasma sheath potential and have to be bent back by particle collisions and the magnetic field to allow extraction through the apertures. A multi-aperture system of several 100 apertures with $8 \mathrm{~mm}$ diameter each is being used. Special formed, chamfered aperture collars are being used to increase the extracted current density [10][9]. The converter surface, which is called plasma grid and the collar design is shown by a schematic diagram of two apertures in figure 1 .

A bias voltage of the plasma grid against the source body is applied in order to suppress the co-extracted electron current. Furthermore, the bias voltage results in a reduction of the sheath potential, an effect that lowers the starting energy of the negative ions. This is advantageous for the transport and extraction process as shown in [9]. The negative ions entering the extraction system through the plasma grid apertures are immediately 

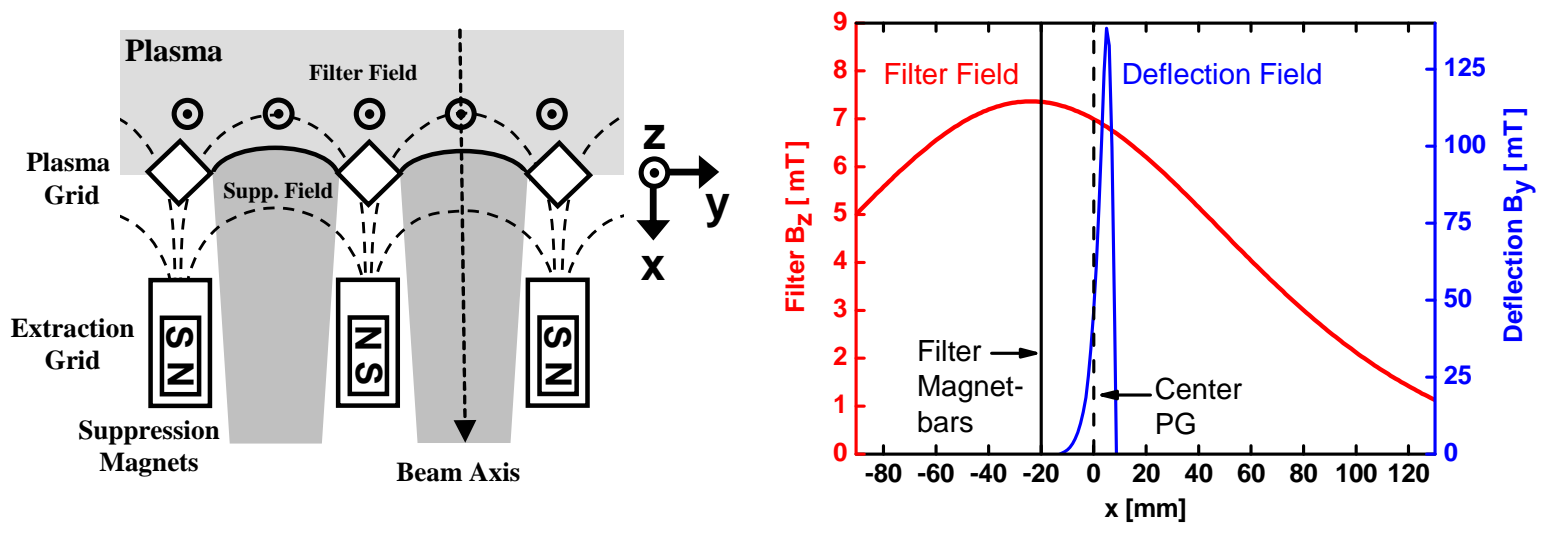

Figure 1. Left: Schematic view of the converter surface, the magnetic filter (z-direction) and the electron suppression field (dashed line). Right: Calculated magnetic field components along an axis of a beamlet (dashed line in the left figure).

accelerated by the extraction voltage.

Ion beam formation is accomplished in two stages by a three grid system: the plasma grid, the extraction grid and the grounded grid. In the first step, a voltage of $8-10 \mathrm{kV}$ is applied between the plasma and the extraction grid in order to allow the removal of coextracted electrons at reasonable energies, which is done by a mass selective magnetic field. This electron suppression field is generated by CoSm magnet rods inside the extraction grid. A second voltage of $15 \mathrm{kV}$ is used for acceleration at the ion source test facility of the MPI für Plasmaphysik.

As stated above, special attention was given to an accurate description of the magnetic field configuration of the extraction region, which will be described in the next section.

\section{Magnetic Field and Converter Topology}

The 3D magnetic topology in the extraction region is given by a superposition of two field components:

(i) filter field in the source body

(ii) electron suppression field from the extraction system

An overview of the converter geometry and the magnetic field lines at the extraction region is given by figure 1. The magnetic filter field is generated by boxes of aligned CoSm permanent magnets attached to the walls of the source body. By this means, a directional field in positive z-direction with $7 \mathrm{mT}$ at the center is generated between the magnets. A contour plot of the field topology is shown in [9].

While the filter field is present in the whole extraction region, the electron suppression field is localized near the plasma grid. The suppression field is generated by permanent magnet rods with alternating magnetization inside the extraction grid. Therefore, the 
direction of the suppression field alternates along the aperture rows and the field lines are orthogonal to the field lines of the filter field. Figure 1 shows the variation of the dominating filter and suppression field components in $\mathrm{x}$-direction. The calculation was done for a line at the center of an extraction aperture (beam axis). The filter field has a Gaussian shaped profile with a peak strength of $7 \mathrm{mT}$ at the position of the magnet boxes at $\mathrm{x}=-20 \mathrm{~mm}$. In contrast, the suppression field reaches it's maximum strength at the position of the extraction grid for $\mathrm{x}=10 \mathrm{~mm}$. Nevertheless, the field penetration into the plasma is limited owing to the alternating magnetization of the magnet rods. In summary, the magnetic field configuration consists of the weak, but long ranging filter field and a strong, but localized suppression field.

\section{Transport Code TrajAn}

The transport code TrajAn has been used to compute the negative ion transport from the converter to the extraction region. A detailed description of the TrajAn code and the applied computational methods is given in [9]. The transport calculation is based on the solution of the 3D Lorentz equation of motion within the magnetic and electric fields of the ion source. Because of quasi-neutrality the electric field within the plasma volume is assumed to be zero except for the extraction fields at the apertures from the extraction system. These fields were calculated self consistently with the Ray Tracing code KOBRA3 [13].

Elastic and inelastic collisions of negative ions with plasma and gas particles are simulated using a Monte Carlo method. The reaction probabilities are computed on the basis of reaction rates for the corresponding particle densities and temperatures. Reactions and plasma parameters, which are specific to the conditions within the described RF-driven negative ion sources, were implemented in TrajAn. Table 1 gives an overview of the reactions, which significantly influence on the Monte Carlo simulation. The symbol H represents either hydrogen or deuterium. Collision cross section data for hydrogen was also applied to deuterium ion transport. An identical set of plasma parameters was used for the transport calculations of negative hydrogen and deuterium ions. These were taken from experimental data at $\mathrm{x}=-2 \mathrm{~cm}$ for source operation with hydrogen described in [11]. Table 2 gives an overview of the plasma parameters used in the transport code.

While spectroscopic investigations in the extraction region of the RF-driven ion source show only weak differences of $\mathrm{T}_{\mathbf{e}}$ and $\mathrm{n}_{\mathbf{e}}$ for hydrogen and deuterium discharges, $\mathrm{a}$ factor of 1.5 higher atomic density $n_{\mathbf{D}}$ was measured in deuterium [11]. Nevertheless, an identical plasma parameter set was used in the transport simulation in order to provide a separation of the individual effects on the ion transport.

A statistics of extracted and destroyed ions is calculated for a large ensemble of particles with different starting coordinates on the converter surface. Surface densities of up to $10^{5}$ homogeneously distributed particles for surface cells of $1 \mathrm{~mm}^{2}$ area were used 
Table 1. Processes taken account in the transport simulation.

\begin{tabular}{lll}
\hline Destructive & & \\
Electron Stripping & $\mathrm{H}^{-}+\mathrm{e} \rightarrow \mathrm{H}+2 \mathrm{e}$ & {$[14]$} \\
Mutual Neutralization & $\mathrm{H}^{-}+\mathrm{H}^{+} \rightarrow 2 \mathrm{H}$ & {$[15]$} \\
& $\mathrm{H}^{-}+\mathrm{Cs}^{+} \rightarrow \mathrm{H}+\mathrm{Cs}$ & {$[16]$} \\
Collisional Detachment & $\mathrm{H}^{-}+\mathrm{H} \rightarrow \mathrm{H}+\mathrm{H}+\mathrm{e}$ & {$[14]$} \\
& $\mathrm{H}^{-}+\mathrm{H}_{2} \rightarrow \mathrm{H}+\mathrm{H}_{2}+\mathrm{e}$ & {$[17]$} \\
Associative Detachment & $\mathrm{H}^{-}+\mathrm{H} \rightarrow \mathrm{H}_{2}+\mathrm{e}$ & {$[14]$} \\
& & \\
Non-Destructive & & \\
Coulomb & & \\
Charge Exchange & $\mathrm{H}^{-}+\mathrm{H}^{+} \rightarrow \mathrm{H}^{+}+\mathrm{H}^{-}$ & {$[18]$} \\
\hline
\end{tabular}

Table 2. Particle densities and temperatures used in the code for both ion species.

\begin{tabular}{lll}
\hline$n_{\mathrm{e}}$ & Electron Density & $5.5 \cdot 10^{17} \mathrm{~m}^{-3}$ \\
$T_{\mathrm{e}}$ & Electron Temperature & $2.0 \mathrm{eV}$ \\
$n_{\mathrm{H}}$ & H Atom Density & $1.0 \cdot 10^{19} \mathrm{~m}^{-3}$ \\
$T_{\mathrm{H}}$ & $\mathrm{H}$ Atom Temperature & $0.8 \mathrm{eV}$ \\
$n_{\mathrm{H}_{2}}$ & $\mathrm{H}_{2}$ Density & $4.0 \cdot 10^{19} \mathrm{~m}^{-3}$ \\
$T_{\mathrm{H}_{2}}$ & $\mathrm{H}_{2}$ Temperature & $1200 \mathrm{~K}$ \\
$\mathrm{H}^{+}$ & $\mathrm{H}^{+}$Ion Density & $5.0 \cdot 10^{17} \mathrm{~m}^{-3}$ \\
$T_{\mathrm{H}^{+}}$ & $\mathrm{H}^{+}$Ion Temperature & $0.8 \mathrm{eV}$ \\
$n_{\mathrm{Cs}^{+}}$ & $\mathrm{Cs}^{+}$Ion Density & $5.0 \cdot 10^{16} \mathrm{~m}^{-3}$ \\
\hline
\end{tabular}

in the simulation considering a complete converter surface of 146 extraction apertures. These densities are necessary to achieve statistical significance in order to resolve spatial profiles of the extraction probability and the current density.

The total or integrated extraction probability is calculated by dividing the number of extracted ions by the number of negative ions, which were altogether started from the converter surface in the simulation. A local extraction probability is defined by counting the number of extracted ions by a specific surface element and dividing it by all particles started from this element.

\section{Results}

\subsection{Integrated Extraction Probability}

The transport calculation was performed for a parameter range of the magnetic field strengths and the starting energies of the surface generated ions. This starting energy 

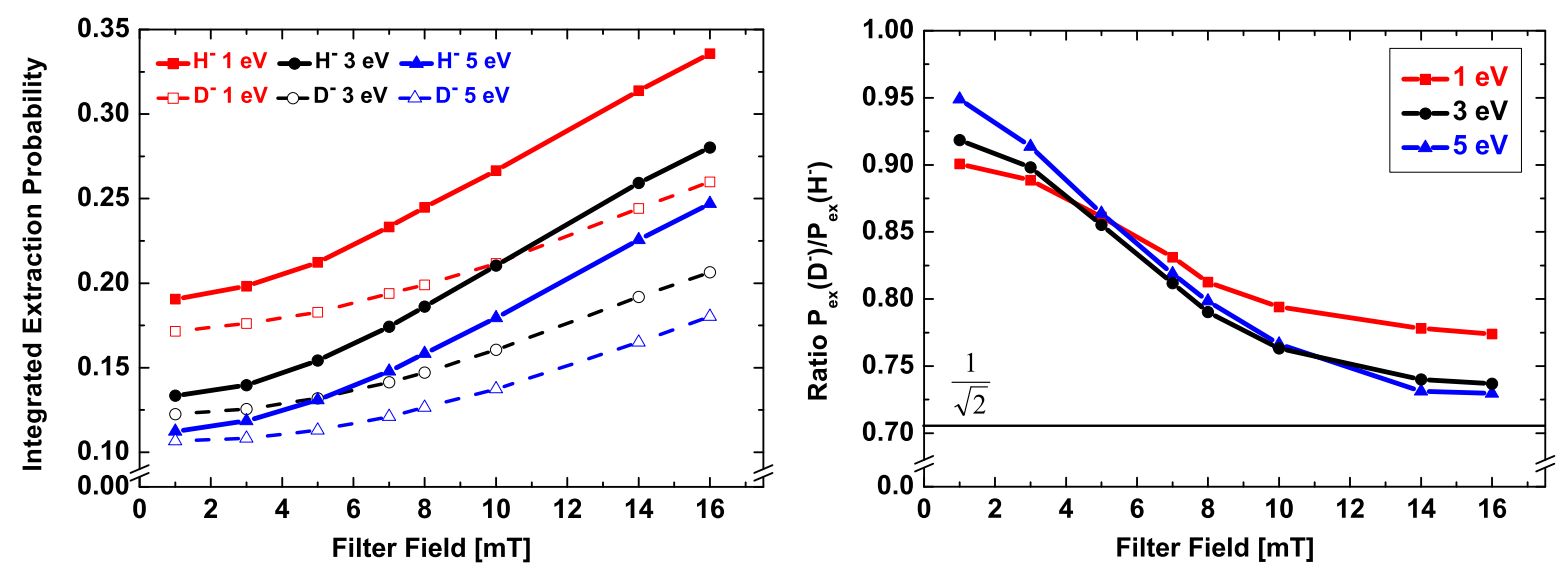

Figure 2. Left: Integrated extraction probabilities of negative hydrogen and deuterium ions versus the magnetic filter strength for 1,3 and $5 \mathrm{eV}$ starting energy. Right: Corresponding ratio of the extraction probabilities. The factor $\frac{1}{\sqrt{2}}$ indicates the mass related scaling ratio of the radii of gyration.

is assumed to correspond to the energy gained by the particle in the sheath prior to the conversion. Calculations of the total extraction probability for hydrogen and deuterium ions were done for the geometry of the extraction system considering a realistic electric and magnetic field configuration. Identical number of surface produced negative ions for both isotopes were used within the scope of the analysis. A parameter scan of the total extraction probabilities was carried out for ion starting energies of 1,3 and $5 \mathrm{eV}$, which represent typical energies for the negative ions in the RF-driven ion source. The strength of the magnetic filter field was scaled for the transport investigation, based on the standard filter field strength of $7 \mathrm{mT}$ at the center of the plasma grid for hydrogen operation. A profile of the main filter field component of this standard configuration along the beam axis is shown in figure 1. Results of this analysis are presented in figure 2 for hydrogen and deuterium ions. The total extraction probability was plotted versus the magnetic field strength for different starting energies. The right figure shows the corresponding ratios of the extraction probabilities of the two species.

A lower total extraction probability was calculated for deuterium over the whole parameter range. The ratio of the extraction probabilities decreases with the filter field strength for all three starting energies. A ratio of 0.73 is reached for a high magnetic field strength and starting energy, which is close to the scaling of the radii of gyration $\mathrm{r}_{\text {gyro }}$ for hydrogen and deuterium ions. Owing to their higher mass, deuterium ion have a lower starting velocity than hydrogen ions for similar particle energies. This effect is especially pronounced for a high filter field strength. The radius of ion gyration has a total scaling of $r_{D^{-}}=\sqrt{2} r_{H^{-}}$and is therefore higher in case of deuterium ions. The increased path length of deuterium ions and the lower particle velocity cause more collisions with the background particles of the plasma volume than in case of hydrogen. 


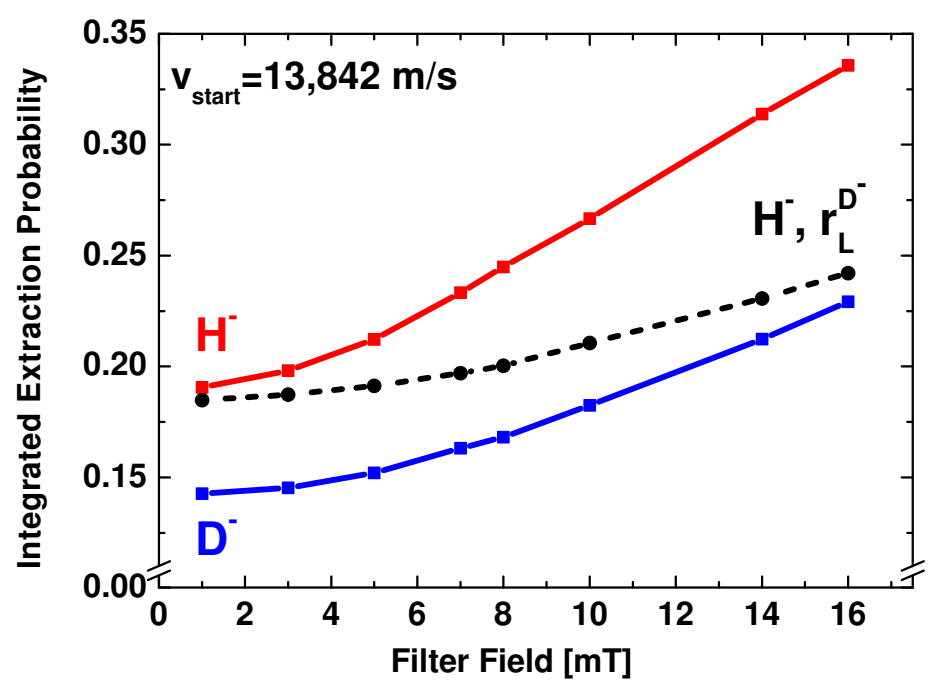

Figure 3. Integrated extraction probabilities of negative hydrogen (red) and deuterium ions (blue) with identical starting velocities corresponding to $1 \mathrm{eV} / \mathrm{amu}$. The dashed curve shows a calculation for hydrogen ions considering the mass of deuterium for the Larmor radius.

In total, this leads to an effective reduction of the extraction probability caused by the increased number of destructive collisions. Nevertheless, the probability for a nondestructive charge exchange collision also increases. It has been shown [9] that this process is beneficial for the extraction probability, related to the redirection of negative ion towards the extraction system. The increase of destructive collisions, however, is the dominating effect in this case.

\subsection{Separation of Mass Related Effects}

Substituting hydrogen with the heavier deuterium at equivalent plasma parameters affects the transport process by three parameters: the starting velocity, the Larmor radius and the mass effect on the collision processes. It has been shown already in [9] that a higher starting velocity results in a reduction of the extraction probability. In order to enable an investigation of the other two effects, the starting velocity of negative hydrogen and deuterium ions was kept constant at 13,842 m/s, which corresponds to a starting energy of $1 \mathrm{eV} / \mathrm{amu}$. Separation of the effect of the Larmor radius and the effect of the ion mass on the collision process was done by performing a transport computation using the mass of deuterium for the effect of the Lorentz force and the mass of hydrogen for the collision force. By this means the effect of the change of the Larmor radius can be separated. The results of this computation are given in figure 3 , which shows the integrated extraction probability for hydrogen/deuterium ions with a starting energy of $1 \mathrm{eV} / \mathrm{amu}$ and the extraction probability for hydrogen with the Larmor radius of deuterium. This analysis shows that the effect of the Larmor radius, which is increased 

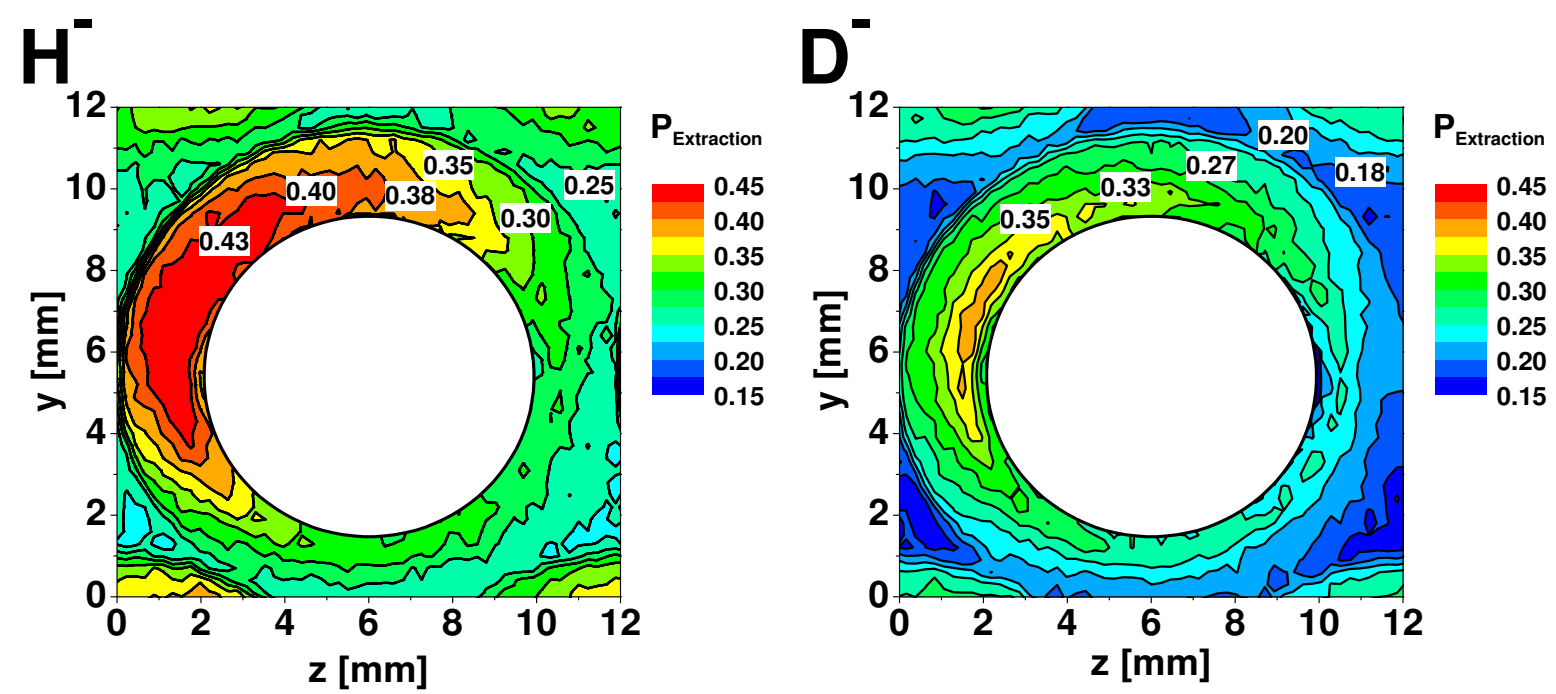

Figure 4. Local extraction probability contour of negative hydrogen and deuterium ions for an aperture at a central position of the plasma grid with filter strength of $7 \mathrm{mT}$. A starting energy of $1 \mathrm{eV}$ was used for both ion species.

in case of deuterium, is less pronounced in the case of a weak magnetic field of $1 \mathrm{mT}$, but gains influence with increasing magnetic field strength. At a strong magnetic field of $16 \mathrm{mT}$ the decrease of the extraction probability in case of deuterium is dominated by the increase of the Larmor radius.

\subsection{Spatial Resolution}

As a result of the complicated structure of the magnetic field and the converter topology, it is necessary to investigate the local distribution of the birthplaces of the extracted ions. This local extraction probability profile was calculated at a central aperture of the converter surface, where a filter field strength of $7 \mathrm{mT}$ is reached.

Figure 4 shows a contour plot of the local extraction probability for $\mathrm{H}^{-}$and $\mathrm{D}^{-}$ions for a starting energy of $1 \mathrm{eV}$. An increase of the local extraction probability was calculated for both species at the aperture collar (2 mm radial distance to aperture), which is a geometric effect due to an advantageous starting angle and the proximity to the extraction aperture. The local extraction probability distribution is affected for both ion species by the orientation of the short range electron suppression field, generated by the magnets inside the extraction grid, which points in y-direction. This shifts the probability distribution in the negative z-direction. The influence of the long range filter field, pointing in z-direction, is also visible in both cases shifting both distributions in the positive y-direction. For deuterium, a lower total extraction probability is visible in the local extraction probability distribution, which corresponds to the results discussed in relation to figure 2 . 

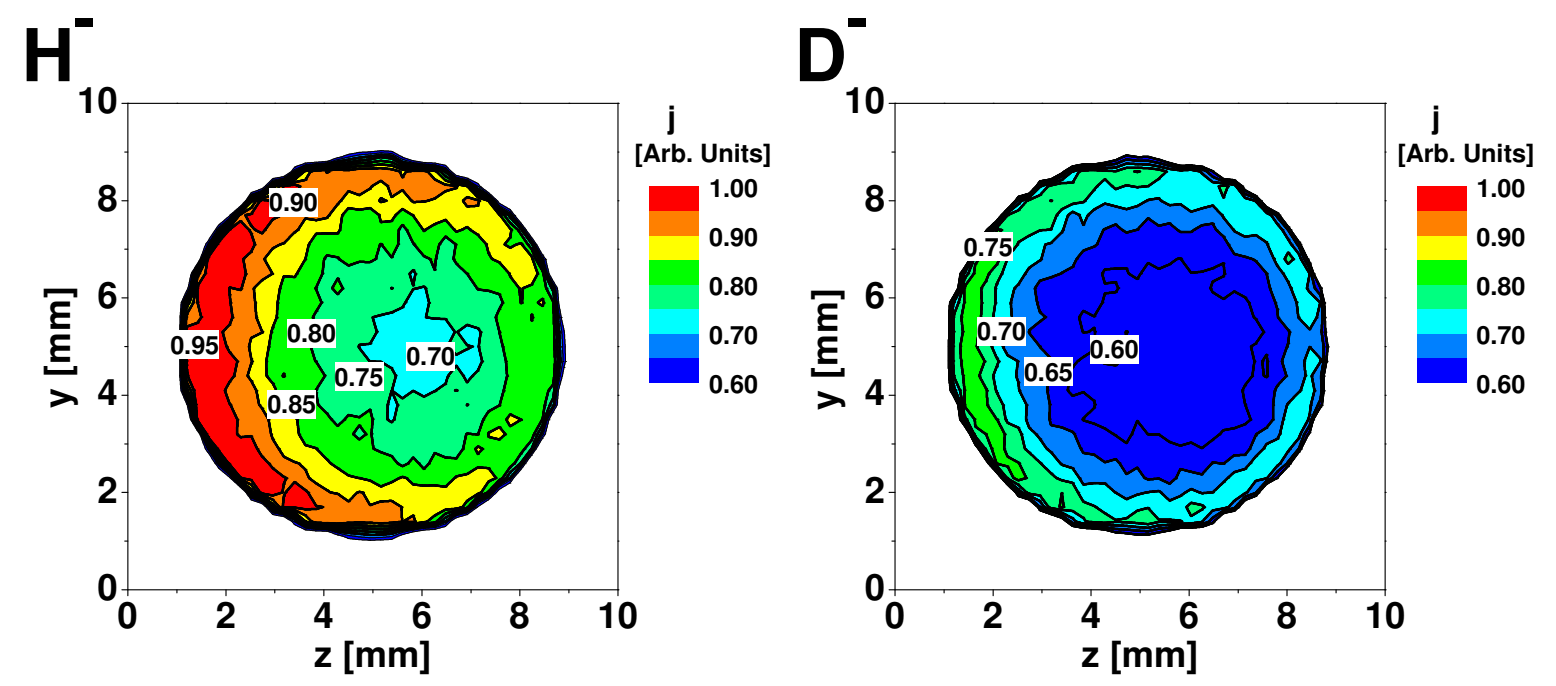

Figure 5. Current densities distribution of negative hydrogen and deuterium ions for an aperture at a central position of the plasma grid with filter strength of $7 \mathrm{mT}$. A starting energy of $1 \mathrm{eV}$ was used for both ion species.

Besides a determination of the local extraction probability, TrajAn is also capable of calculating a distribution of the current density over an aperture. This is done by integrating the number of extracted ions at an area element of the plasma-beam transition surface. Results for the distribution of deuterium and hydrogen are presented in figure 5. A lower current density is obtained for deuterium ion extraction, which is in agreement with results from the extraction probability. In both cases, the transport calculation shows that the current density is concentrated near the aperture edge. This effect is related to the shorter mean path length upon extraction at this starting positions.

Both species react sensitively on the suppression field component and the corresponding birth and extraction distributions show a inhomogeneity. Calculations for hydrogen and deuterium ions show a shift of the current density distribution according to the local orientation of the magnetic field. This effect is very similar to the effect in the local extraction probability.

\section{Conclusion}

Probabilistic ion transport calculations for surface generated hydrogen and deuterium ions were performed for high power, large area negative ion sources designated for the ITER neutral beam injection. An overall lower extraction probability for deuterium ions was calculated for the case of identical plasma parameters and field conditions.

An investigation of local extraction probability and current density profiles showed asymmetric birth and current density distributions primarily generated by the short 
range suppression field in case of both ion species.

Experimental data of hydrogen and deuterium operation in the RF-driven ion source show a similar source efficiency for both species [11]. Therefore the decreased extraction probability from the transport calculation in case of deuterium has to be (over)compensated by a different effect, i.e. by different plasma parameters for deuterium. One of the next steps will be the implementation of future, more detailed measurements of the plasma parameters for deuterium operation.

\section{Acknowledgments}

This work was (partly) supported by a grant from the European Union within the framework of EFDA (European Fusion Development Agreement). The authors are solely responsible for the content.

\section{References}

[1] E. Speth et al., Fus. Eng. Des. 46 (1999), 383

[2] K.H. Berkner, R. V. Pyle and J. W. Stearns, Nucl. Fus. 15 (1975), 249

[3] R. S. Hemsworth, A. Tanga and V. Antoni, Rev. Sci. Inst. 79 (2008), 02C109

[4] ITER Technical Basis 2002, ITER EDA Documentation Series No 24 (Plant Description Document, section 2.5.1), IAEA Vienna

[5] B. Heinemann, H. D. Falter, U. Fantz, and P. Franzen et al., Fus. Eng. Des. 84 (2009) 915922

[6] D. Wünderlich, R. Gutser and U. Fantz, Plasma Sources Sci. Technol., submitted

[7] O. Fukumasa and R. Nishida, Nucl. Fusion 46 (2006), 275

[8] D. Riz and J. Pamela, AIP Conference Proceedings 380 (1996), 3

[9] R. Gutser et al., Plasma Phys. Control. Fusion 51(2009), 045005

[10] E. Speth, H. Falter, P. Franzen, U. Fantz et al, Nucl. Fusion 46 (2006), 220

[11] U. Fantz U et al, Nucl. Fusion 46 (2006), 297

[12] W. G. Graham, "Properties of Alkali Metals Adsorbed onto Metal Surfaces" in Proc. 10th Symp. on Fus. Tech., Brookhaven National Laboratory, Upton, 1980

[13] P. Spädtke and S. Wipf, GSI Report 89-09 (1989)

[14] R. Janev, W. Langer, J. Evans and D. Post, "Elementary Processes in Hydrogen-Helium Plasmas", Springer, Berlin, 1987

[15] M. Eeerden, M. van Sanden, D. Otorbaev and D. Schram, Phys. Rev. A 51 (1994), 3362

[16] R. Janev and Z. Radulovic, Phys. Rev. A 17 (1978), 889

[17] C. F. Barnett, Oak Ridge National Laboratory, Technical Report ORNL-6086, 1990.

[18] S. Ma, R. Sydora and J. Dawson, Comp. Phys. Comm. 77 (1993), 190

[19] M. Huels, R. Champion, L. Doverspike and. Y. Wang, Phys. Rev. A 41 (1990), 4809 